\title{
TOWARDS A DOMAIN-RELEVANT APPROACH TO THE EVOLUTION OF LANGUAGE AND MUSIC
}

\author{
RIE ASANO*1 \\ ${ }^{*}$ Corresponding Author: rie.asano@uni-koeln.de \\ ${ }^{1}$ Department of Systematic Musicology, University of Cologne, Cologne, Germany
}

The central idea of current comparative research on the evolution of language and music is that they consist of multiple components with different evolutionary origins (Fitch, 2006). From a comparative language-music perspective, some components might be shared and based on the same evolutionary genesis, while others might be different and emerged independently in the course of evolution. From a comparative between-species perspective, some might be shared with other animals, while others might be unique to humans. However, this shareddistinct dichotomy dominating the recent comparative approach usually depends on tailor-made categories fitting to just one domain or species and thus limits the range of investigation by its all-or-nothing contrastive view (De Waal \& Ferrari, 2010; Theofanopoulou \& Boeckx, 2015).

To go beyond such a shared/distinct dichotomy, the current paper puts a domain-relevant approach forward. Its main idea is that through neural competition, brain networks become relatively domain-specific overtime (Karmiloff-Smith, 2013). Thus, specialization of function can be regarded as fine tuning of coarsely coded systems with domain-relevant biases. In evolutionary research, those domain-relevant systems should be the target of comparative endeavor investigating the evolution of language and music. Based on evidence from cognitive and evolutionary neuroscience (Kotz et al., 2009; Lieberman, 2016; Merchant et al., 2015; Ullman, 2006) as well as modeling studies (e.g., Dominey et al., 2009), I suggest that the cortico-basal ganglia-thalamocortical (CBGT) circuits form a promising candidate for such systems.

The CBGT circuits are involved in and are necessary for performing a procedure, i.e. organizing sequences of actions towards a goal. For example, syntactic sequence processing in language and beat-based rhythmic sequence 
processing in music build on those circuits. They are also required for procedural learning such as habit and rule learning. Impairments of the CBGT circuits result in underspecified rule representations in language and music. Therefore, from a neurocognitive perspective the cognitive systems language and music can be regarded as a different use of the same domain-relevant systems.

Moreover, investigations of the CBGT circuits provide direct betweenspecies comparative options: those circuits are required for non-human primates' action cognition (Mendoza \& Merchant, 2014) as well as song learning in songbirds (Jarvis, 2004). Thus, the current paper provides strong support for hypotheses that regard both these current neurocognitive systems as products of evolutionary changes of an ancestral action cognition systems (Boeckx \& Fujita, 2014; Fujita, 2016; Marcus et al., 2006).

\section{Acknowledgements}

Special thanks to Prof. Uwe Seifert for his helpful comments to the earlier version of this abstract.

\section{References}

Boeckx, C., \& Fujita, K. (2014). Syntax, action, comparative cognitive science, and Darwinian thinking. Frontiers in Psychology, 5, 627.

De Waal, F. B. M., \& Ferrari, P. F. (2010). Towards a bottom-up perspective on animal and human cognition. Trends in Cognitive Sciences, 14(5), 201-7.

Dominey, P. F., Inui, T., \& Hoen, M. (2009). Neural network processing of natural language: II. Towards a unified model of corticostriatal function in learning sentence comprehension and non-linguistic sequencing. Brain and Language, 109(2-3), 80-92.

Fitch, W. T. (2006). The biology and evolution of music: a comparative perspective. Cognition, 100(1), 173-215.

Fujita, K. (2016). On the parallel evolution of syntax and lexicon: A Merge-only view. Journal of Neurolinguistics, 1-15.

Jarvis, E. D. (2004). Learned Birdsong and the Neurobiology of Human Language. Annals of the New York Academy of Sciences, 1016(1), 749-777.

Karmiloff-Smith, A. (2013). Challenging the use of adult neuropsychological models for explaining neurodevelopmental disorders: developed versus developing brains. Quarterly Journal of Experimental Psychology, 66(1), 114.

Kotz, S. A., Schwartze, M., \& Schmidt-Kassow, M. (2009). Non-motor basal ganglia functions: A review and proposal for a model of sensory predictability in auditory language perception. Cortex, 45(8), 982-990. 
Lieberman, P. (2016). The evolution of language and thought. Journal of Anthropological Sciences, 94, 1-20.

Marcus, G. F., Rabaglia, C. D., \& Rabagliati, H. (2006). Modularity and descentwith-modification. In C. Boeckx \& K. K. Grohmann (Eds.), The Cambridge Handbook of Biolinguistics (pp. 326-340). Cambridge: Cambridge University Press.

Mendoza, G., \& Merchant, H. (2014). Motor system evolution and the emergence of high cognitive functions. Progress in Neurobiology, 122, 73-93.

Merchant, H., Grahn, J. A., Trainor, L., Rohrmeier, M., \& Fitch, W. T. (2015). Finding the beat: a neural perspective across humans and non-human primates. Phil. Trans. R. Soc. B, 370, 20140093.

Theofanopoulou, C., \& Boeckx, C. (2015). Cognitive phylogenies, the Darwinian logic of descent, and the inadequacy of cladistic thinking. Frontiers in Cell and Developmental Biology, 3(October), 1-6.

Ullman, M. T. (2006). Is Broca's Area Part of a Basal Ganglia Thalamocortical Circuit? Cortex, 42(4), 480-485. 